\title{
On thermal emission of small-sized radiator
}

\author{
E.A. Salkov, G.S. Svechnikov \\ Institute of Semiconductor Physics, NAS of Ukraine, 45 prospect Nauky, 03028 Kyiv, Ukraine
}

\begin{abstract}
Principle feasibility has been considered for a distant identification of a small-sized thermal radiator by means of detecting its thermal radiation. A single small-size radiator is phenomenologically treated within the black body model. It is shown to be possible to obtain a numeral evaluation of the temperature and the size of a small-size radiator through measurement of the thermal radiation fluctuations in case when optical image of the radiator is unavailable.
\end{abstract}

Keywords: black body, thermal radiation, size-restrictions, fluctuations, variance.

Paper received 19.05.03; accepted for publication 16.06.03.

\section{Introduction}

Nowaday technology of distant identification of largesize emitters of thermal radiation (TR) has been developed almost perfectly. As regards TR radiators of a very small size(comparable with the wavelength of observation), the problem is accentuated by fundamental optical and technical restrictions.

Analogous problem was previously considered in connection with the spectroscopy of the Moon surface [1] . Thermodynamic analysis of TR inside limiterd-size black body (b.b.) was developed in [2 ]. Contribution of the size-factor to the b.b.TR spectrum can be approximately taken into account by deducting of three lowest equilibrium modes from their whole number [ 3 ]. The size-factor for the small-sized radiator ( SSR ) of a cubic form appears in the form of the multiplier[ 2,3 ]

$$
\left[1-\frac{\lambda_{o b s}^{2}}{4\langle V\rangle^{2 / 3}}\right]
$$

where $\lambda_{o b s}$ is the observed TR wavelength from the SSR of a cavity mean volume $\langle V\rangle$. The term "small-sized radiator" in our case is determined by the commensurability of the SSR linear size $R$ and the wavelength of TR radiation. For mathematical convenience the b.b. configuration is considered in a form that allows the approximate equality $R \approx\langle V\rangle^{1 / 3}$.

On the other hand, the possibility seems to be realisable to conjure parameters of the SSR from the data of the TR fluctuations. Regarding the above problem we will employ the "micro quantity of chaos" (MQC) concept $[4,5]$. The MQC treated in this paper is a physically defined intrinsic micro-parameter $q(F)$ of stochastic system. Being an essential intrinsic microscopic quantity $q(F)$ directly determines the variance $\left\langle\Delta F^{2}>\right.$ of a macroscopic physical quantity $F$ via its mean value $\langle F\rangle$ in accordance with the known formula

$$
<\Delta F^{2}>=q(F) \times<F>
$$

The validity of the $q(F)$ concept as the entirely defined physical quantity has been proved in series of statistical situations e.g. for the ideal gas, thermal black 


\section{E.A. Salkov, G.S. Svechnikov: On thermal emission of small-sized radiator}

body radiation, electrical and photocurrents [4,5]. Incontrovertible candidate for this rule is Poisson's statistic of uncorrelated processes. For further calculations we will use the following expressions of the intrinsic $q(\mathrm{TR})$ for the black body TR[ 5$]$ :

$q(E)=\frac{h C}{\lambda} \cdot(1+<n>)-$ intrinsic TR energy

$q(P)=\frac{h C}{\lambda} \cdot(1+<n>) \cdot \Delta v_{p}-$ intrinsic TR power,

where $\langle n\rangle=[\exp (h c / \lambda k T)-1]^{-1}$ is Planck's function or Bose-Einstein statistic distribution, $C$ is the velocity of light, and $\Delta v_{p}$ is an intrinsic frequency bandwidth within a certain $q(E)$ acts. Determination of $\Delta v_{p}$ is a procedure of decisive importance in consideration of SSR TR power. Note, that in our earlier papers the $\Delta v_{p}$ quantity was not defined[ 4, 5 ].

The approach proposed here is not a rigorous one, this is rather a sketch of the phenomenon. The treatment performed on the base of simplified model [ 3 ] of the small size b.b. is intended to reveal the general features of the problem and feasible approaches to the solution.

\section{Size-restricted black body thermal emission}

\subsection{TR energy of a small-size b.b. within a narrow frequency band $\Delta v$.}

Below we will use the expression for the b.b. TR spectrum in the form based on the supposition that the ratio $|\Delta v / v|=|\Delta \lambda / \lambda|$ is keeping fixed during the measurement of the spectrum. The last eliminates "conflict" situation between reciprocal scales of " $v$ " and " $\lambda$ " for a specified photon parameters. Thus, the spectral distribution of TR energy of the "small-size black body" of volume $V \approx R^{3}$ can be written in the form

$$
E_{V}(\lambda) \cong 8 \pi \cdot R^{3} \frac{h C}{\lambda^{4}}\left[1-\frac{\lambda^{2}}{4 R^{2}}\right]\left(\frac{\Delta \lambda}{\lambda}\right)<n>
$$

and, the dispersion is ( see e.g. [ 6, 7, 8 ])

$$
<\Delta E_{V}^{2}>=<E_{V}(\lambda)>\rtimes\left[\frac{h C}{\lambda}(1+<n>)\right] .
$$

At $\lambda_{\text {obs }}<<2 R$ Eqs. $(4,5)$ are transformed into the well known classic form, but when $\lambda_{\text {obs }} \rightarrow 2 R$ the $S S R$ as the black-body disappears from the field of view.

Note, that the quantities $\left\langle E_{V}(\lambda)\right\rangle$ and $\left\langle\Delta E_{V}^{2}\right\rangle$ can not be measured directly. In practice they are determined only through the detected photon power flows exciting a photodetector (PD). To gain further insight it is interesting to consider fluctuations of the photons power flows inside the small-size b.b. cavity.

\subsection{SSR TR power within a narrow frequency band $\Delta v$}

The most general interrelation between energy and power is defined via the ratio (energy/time $) \equiv($ energy $\times$ frequency band ). This time (or frequency ) interval can be extracted in the evident form by means of expression of TR power via the total TR energy $E_{V}(\lambda)$, Eq. ( 4 ) inside the SSR cavity. In case of an ideal "radiator- detector" path (losses are equal to zero), TR power $P_{V D}$ that excites PD do not depend on physical parameters of this path. Thus the TR photon flow which outflows from area $A$ of a single radiator squarely to its surface, within a small solid angle $\Omega \cong D^{2} / L^{2}$, excites an ideal PD(with an area $D^{2}$ ) can be expressed via the total b.b. TR energy $E_{V}(\lambda)$ as

$F_{V D}=\frac{E_{V}(\lambda)}{h C / \lambda} \cdot\left[\frac{C}{2 R}\right] \cdot \frac{A}{R^{2}} \cdot \Omega$.

It is evident that there is only one explicit frequency (time) parameter $\Delta v_{P}=[C / 2 R]$ which connects power with the energy ( 4$)$. We will discuss the value of $\Delta v_{P}$ in details later on.

As an example, Fig. 1 exhibits an adequate to Eqs. 4 and 6 normalized spectra of TR photon flows as functions of the radiator size.

As can be seen, the most significant deviation from the classic TR spectrum take place in a longwave region, especially when the wavelength $\lambda \rightarrow 2 R$. A general profile of the SSR TR spectral distribution provides initial information about the size of the SSR. For the SSR $\lambda_{\max }$ is shifted towards the shorter wavelength in comparison with large-size radiator or classic b.b. Therefore the temperature displayed by the SSR was higher than actual temperature. Nevertheless, some numeral evaluations can be obtained from the TR spectra.

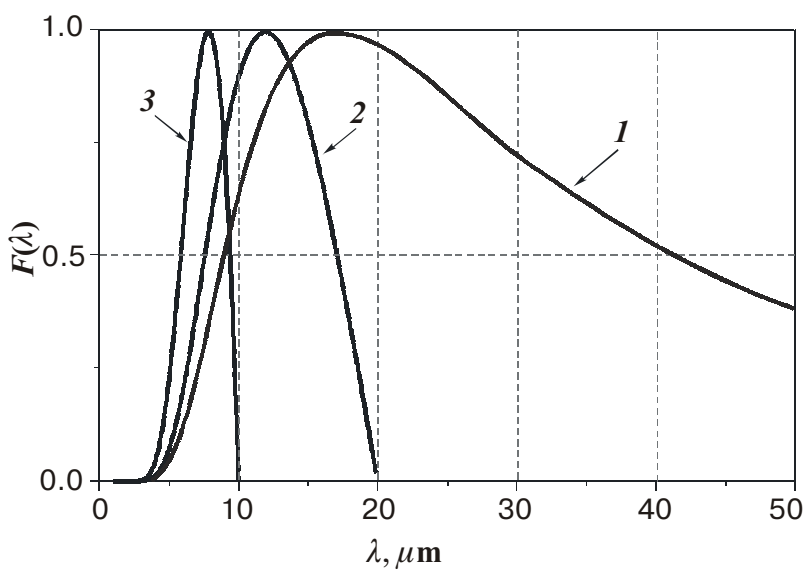

Fig. 1. Normalized spectra of TR photon flows from the radiators of various sizes. $T=300{ }^{0} \mathrm{~K} . I-$ large-size radiator $R \geq 10 \mathrm{~cm}$; 2 - small-size radiator $R=10 \mu \mathrm{m} ; 3$ - small-size radiator $R=5 \mu \mathrm{m}$. 


\section{E.A. Salkov, G.S. Svechnikov: On thermal emission of small-sized radiator}

\section{TR power fluctuations at the photodetector input}

\author{
3.1. Bandwidth $\Delta v_{p}$ for the SSR TR power \\ inside the cavity.
}

As the intrinsic TR energy $q(E)=\frac{h C}{\lambda} \cdot(1+<n>)$ from Eq. ( 2 ) has been firmly established [ 4,5$]$ in agreement with universally recognised references [ e.g. 6, 7, 8 ], it seems to be logical to assume the existence of the intrinsic quantity $q\left(P_{V}\right)$ that is adequate to the $q(E)$ and related to the b.b.TR power inside the cavity of volume $V$.

The main problem now is how to define the bandwidth $\Delta v_{p}$ or proper spectral sampling time $T_{t r}$ which determines $q\left(P_{V}\right)$ via the $q(E)$. This problem arises from the indubitable fact that the optical bandwidth of observation $\Delta v$ cannot be fasten to the intrinsic physical processes inside b.b. cavity. Moreover, it is necessary to emphasise that the use of the bandwidth $\Delta v$, (that corresponds to $\Delta \lambda$ in Eq. (6)) in the capacity of the $\Delta v_{p}$ from Eq. (3) is not an adequate act. In fact, value of $\Delta v$ determines number of observed TR modes [6, 7]. The b.b. TR fluctuations under consideration are originated completely inside the b.b. cavity, whereas $\Delta v$ bandwidth is completely controlled by the external observer. Recall also, that photons do not interact among themselves outside the cavity [9].

The intrinsic $q(E)$ "produces" an adequate "virtual intrinsic microscopic power" $q\left(P_{V}\right)=q(E) \cdot \Delta v_{P}$ within the boundaries of the intrinsic frequency band $\Delta v_{p}$. Hence, it seems reasonable to express $\Delta v_{p}$ in terms of a maximum long time interval $T_{t r}$ during of which the $q(E)$ "dissipates" this virtual smallest power $q\left(P_{V}\right)$. If we assume that $T_{t r}$ is equal to the photon one-path transit time through the small-size cavity $T_{t r}=R / C$ and taking into account the well known interconnection between frequency bandwidth and corresponding sampling time in the form of $2 \Delta v_{p}=T_{t r}^{-1}$ [10], evidently, that "internal" time parameter $\Delta v_{p}=C / 2 R$ and hence, $q\left(P_{V}\right)$ inside the b.b. cavity is determined by the frequency band that coincides with time parameter in Eq. (6). Thus, Eq. (3) leads to the following expression of $q\left(P_{V}\right)$ inside the black body cavity:

$q\left(P_{V}\right)=\frac{h C}{\lambda} \cdot(1+<n>) \cdot \frac{C}{2 R}$.

As the b.b. TR fluctuations are originated completely inside the b.b. cavity one may write down the dispersion of $P_{A}$ out off the large-size b.b. cavity in accordance with Eq. (1) as

$<\Delta P_{A}^{2}>=<E>\cdot \frac{C}{2 R} \cdot \frac{A}{R^{2}} \cdot \Omega \cdot\left(\frac{h C}{\lambda}(1+<n>) \cdot \frac{C}{2 R}\right)$

The last equation includes the intrinsic power characteristic Eq. (7). In case of a very small radiator and approximate equality $A \cong R^{2}$, Eq. (8) transforms in to the dispersion of the TR flow at the PD input

$<\Delta F_{A D}^{2}>=\frac{<E_{V}>}{h C / \lambda} \cdot \frac{C}{2 R} \cdot \Omega \cdot\left((1+<n>) \frac{C}{2 R}\right) \cdot \Omega$

To realize the above speculations we have to consider a photocurrents at the output of the PD.

\section{Photodetector currents}

We consider TR detection by means of an ideal PD that gives us the chance to deal with only two kinds of noises: steady-state photocurrent shot noise $<i_{s n}^{2}>[11]$, and chaotic photocurrent $\left\langle i_{n P}^{2}\right\rangle$ from self-fluctuation of photon flow at the PD input [ 12 ]. The latter will be of interest because the $q\left(P_{V}\right)$ (7) depends on the size of the

b.b. cavity via the unique time characteristic $\left[\frac{C}{2 R}\right]$. Just this point clears the way to extract the size and the temperature of the SSR from the TR fluctuations.

From the above suppositions the total PD current is given by the sum of the contributions:

1. Steady state mean photocurrent

$<I_{D}>=e \eta<F_{A D}>$

which is proportional to the mean photon flow striking the PD.

2. Time dependent stochastic photocurrent oscillations or the shot noise conditioned by the steady-state photocurrent

$<i_{s n}^{2}>=2 e<I_{D}>\Delta f=2 e^{2} \eta<F_{A D}>\cdot \Delta f$

where $\eta$ is the PD efficiency (we will assume it to be equal to unit), $e-$ is the electron charge, $\Delta f$ is the bandwidth of the PD electronics that measures chaotic photocurrent.

3. Time depending chaotic photocurrent from selffluctuation of the photon flow at the PD input:

This component is expressed via the dispersion of $<F_{A D}>$, i.e. Eq. (9), as [ 12 ]

$<i_{n P}^{2}>\cong e^{2} \times<\Delta F_{A D}^{2}>\cdot\left(2 \frac{\Delta f}{\Delta v}\right)$

The multiplier $(2 \Delta f / \Delta v)$ in Eq.(12) appears as the result of detailed analysis of the spectral density of the output chaotic PD-current [12] and follow-up integrating of this spectrum to calculate the dispersion connected with the second noise component $\left\langle\Delta F_{A D}^{2}\right\rangle$. Speaking in images, this multiplier is part of the input power fluctuation which is really registered within the bandwidth $\Delta f$ of the PD output chaotic current. Taking into account Eqs. (11) and (12) the summarised dispersion of the output currents is given by 


$$
\begin{aligned}
& <I_{P D}^{2}>=2 e^{2}<F_{V D}>\cdot \Delta f+e \cdot<F_{V D}>\times \\
& \times\left((1+<n>) \frac{C}{2 R}\right) \Omega \cdot\left(2 \frac{\Delta f}{\Delta v}\right)
\end{aligned}
$$

Then, simple algebra leads to the expression of the total quantity of photocurrent MQC

$$
\begin{aligned}
& q_{\text {tot }}=\frac{<I_{P D}^{2}>}{<I_{D}>}=2 e \Delta f \times \\
& \times\left[1+\left(\frac{\exp (h C / \lambda k T)}{\exp (h C / \lambda k T)-1} \cdot\left(\frac{\lambda}{\Delta \lambda}\right) \cdot \frac{\lambda}{2 R} \cdot \Omega\right)\right]
\end{aligned}
$$

Determination of size and temperature of the $S S R$ in general is somewhat cumbersome but quite obtainable. This approach comprises measurements of $q_{\text {tot }}$ at a two different wavelengths $\lambda_{1,2}$ to obtain corresponding variants of Eq.(14). These measurements lead to a pair of quantities, $Q_{1}$ and $Q_{2}$, from which one can get the solvable relations between the directly measured data $q_{1,2}$, predetermined, known quantities $2 e \Delta f, \frac{\Delta \lambda}{\lambda}$ and unknown desired quantities $X=2 R$ and $T$ :

$$
Q_{1,2}=\left[\left(\frac{q_{1,2}}{2 e \Delta f}-1\right) \cdot \frac{\Delta \lambda}{\lambda}\right]=\left(\frac{\lambda_{1,2}}{X}\right) \cdot\left(\frac{\exp \frac{1.44}{\lambda_{1,2} \cdot T}}{\exp \frac{1.44}{\lambda_{1,2} \cdot T}-1}\right)
$$

These expressions for $Q_{1}$ and $Q_{2}$ can be re-arranged in the form

$$
\exp \frac{1.44}{\lambda_{1,2} \cdot T}=\frac{Q_{1,2}}{Q_{1,2}-\frac{\lambda_{1,2}}{X}}
$$

that allows to exclude the SSR temperature by means of taking the logarithm with follow-up dividing of the results. Denoting $\lambda_{2} / \lambda_{1}=m$ we come to the next form of the solvable equation

$$
m \cdot \ln \frac{X}{\left(X-\frac{\lambda_{2}}{Q_{2}}\right)}=\ln \frac{X}{X-\frac{\lambda_{1}}{Q_{1}}} .
$$

Exponentiating Eq. (17) and by using the binomial formula one obtains the polynomial of " $m-1$ " degree with respect to $X$ :

$$
\begin{aligned}
& X^{m-1}+\frac{m(m-1)}{2 !} X^{m-2} \times \\
& \times \frac{\left(\frac{\lambda_{2}}{Q_{2}}\right)^{2}}{\left[\left(\frac{\lambda_{1}}{Q_{1}}\right)-m\left(\frac{\lambda_{2}}{Q_{2}}\right)\right]}-\frac{m(m-1)(m-2)}{3 !} X^{m-3} \times
\end{aligned}
$$

$$
\begin{aligned}
& \times \frac{\left(\frac{\lambda_{2}}{Q_{2}}\right)^{3}}{\left[\left(\frac{\lambda_{1}}{Q_{1}}\right)-m\left(\frac{\lambda_{2}}{Q_{2}}\right)\right]}+\ldots+(-1)^{n} \cdot \frac{m(m-1) \ldots(m-n+1)}{n !} \times \\
& \times X^{m-n} \cdot \frac{\left(\frac{\lambda_{2}}{Q_{2}}\right)^{n}}{\left[\left(\frac{\lambda_{1}}{Q_{1}}\right)-m\left(\frac{\lambda_{2}}{Q_{2}}\right)\right]}+\ldots+ \\
& +(-1)^{m} \cdot \frac{\left(\frac{\lambda_{2}}{Q_{2}}\right)^{m}}{\left[\left(\frac{\lambda_{1}}{Q_{1}}\right)-m\left(\frac{\lambda_{2}}{Q_{2}}\right)\right]}=0
\end{aligned}
$$

The polynomial (18) is solvable by the standard method [13,14].

As example the graphic representation of Eq. (18) solutions $\Psi(R)$ for three different values of ratio $m$ and various temperatures are depicted in Fig. 2.

The exact match of the solutions for the given values $m$ take place at a maximum magnitudes of roots $R=10 \mu \mathrm{m}$. As size $R$ has been evaluated from Eq. (18) one can turn back to Eq. (16) to calculate the SSR temperature $T$.

Note, that the refinement of the problem in case of TR emission by a material substance but not a b.b. can be realized using formulas from e.g., Ref.[6] .

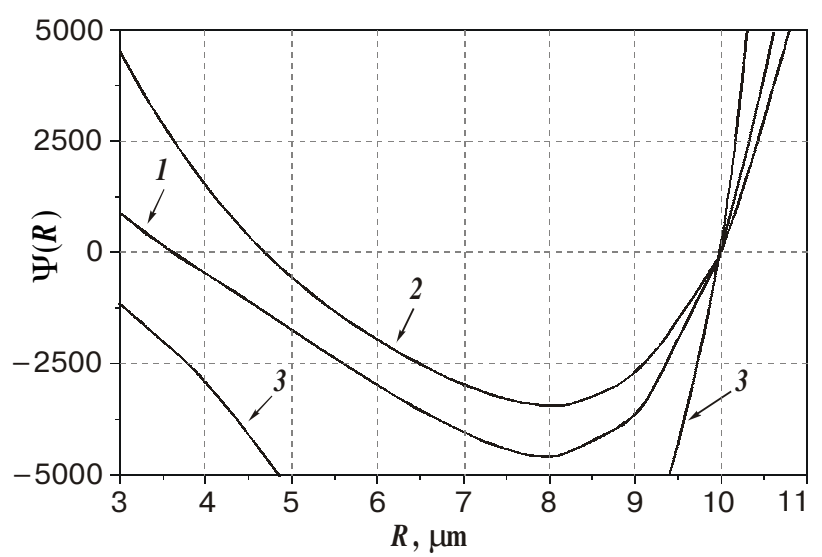

Fig. 2. Graphic solution of Eq. (18) for three different values of ratio $\mathrm{m}$. Predetermined size of SSR $-\mathrm{R}=10 \mu \mathrm{m} . l-m_{1}=10 / 2$, $T=1000{ }^{0} \mathrm{~K}, 2-m_{2}=5 / 1, T=1000{ }^{0} \mathrm{~K}, 3-m_{3}=15 / 3, T=3000{ }^{0} \mathrm{~K}$. 


\section{E.A. Salkov, G.S. Svechnikov: On thermal emission of small-sized radiator}

\section{Conclusion}

In the frames of the above phenomenological consideration the concept of TR dispersion gains certain cognitive contents being directly connected with the physical micro-parameters of radiating system. Especially attractive appears possibility to obtain the SSR parameters via TR fluctuation in case when optical image of the radiator is unavailable.

Moreover we would like to stress a few points:

1) The spectral distribution of the TR provides an approximate data about the SSR size (Fig. 1). This information allows to discriminate a large-size solid TR radiator from a large-size, dense cloud of SSRs.

2) In case of a single SSR simultaneous measurement of $\left\langle I_{D}\right\rangle$ Eq. (10), $<\Delta i_{s n}^{2}>$ Eq.(11) and $<i_{n P}^{2}>$ Eq. (12) determine the value of $q_{t o t}$ which is directly connected with the size and the temperature of radiator. It is necessary to underline the ultimate simplicity of this connection (Eqs.(7), (14)).

3) Measurement of the same quantities $\left\langle I_{D}\right\rangle$, $\left\langle\Delta i_{s n}^{2}\right\rangle$ and $\left\langle i_{n P}^{2}>\right.$ for a single $S S R$ at two different wavelengths make it possible to determine the temperature and the size of the radiator by means of solving Eq.(18). It is a substantial fact that the exponent of the polynomial Eq.(18) power $m=\lambda_{2} / \lambda_{1}$ can be chosen by observer in connection with the concrete situation and results can be tested via picking a new value of $m$.

4) In practice there are probably a number of opportunities to apply the MQC concept [4,5] (in our case it is $q_{\text {tot }}$ ) to convert a "noisy glitch" into a "source of an addition information about the internal properties of the stochastic physical system".

The above approach is valid if only one deals with a single SSR, e.g. element of a large scale integrated circuit(LSIC). But in case of natural aggregation of the SSRs, to derive the dispersion of TR power it is necessary to take into account fluctuation of the SSR number $N_{S}$ within the observation field of view $\Omega_{D}$. This analysis will be performed in our next paper.

\section{References}

1. Salisbury J.W., Glaser P.E., The Lunar Surface Layer, Academic.Press, New York, 1964;

2. A.A.Kolokolov, G.V.Skrotchki, Ravnovesnoe izluchenie v polosti konechnogo razmera.//Optics and Spectroscopy, 36, N.2, pp.217-221.1974.

3. E.A.Salkov, G.S.Svechnikov, Size-restrictions of radiation detecting using semiconductor photodetectors. // Proc.SPIE, 2894, pp. 66-73, 1996.

4. E.A. Salkov Intrinsic "quantity of chaos" in photodetecting.// Proc.SPIE, 3182, pp. 78-84, 1997.

5. E.A. Salkov Microscopic Parameters of Stochastic System and Variance of Physical Quantity (ideal gas, electric currents, thermal black body radiatio. //Semicond. phys.,quant. electronics \& optoelectronics. 1, pp.116-120, 1998.

6. C. V. Heer. Statistical Mechanics, Kinetic Theory, and Stochastic Processes, Academic Press, New York, London, 1972.

7. L.D. Landau, E.M. Lifshic, Statisticheskaya fizika (Teoreticheskaya fizika 5), Moskva, Nauka,1964.

8. H.Schilling: Statistische Physik in Beispielen.VEB Fachbuchverlag Leipzig, 1972

9. P. Dirak, Principi kvantovoi mehaniki. Moskva, Nauka, 1979.

10. Bracewell R. N. : The Fourier Transform and Its Applications, McGraw-Hill, New York 1965.

11. Albert van der Ziel. NOISE . Sources, Characterisation, Measurement. Prentice -Hall ,Englewood Cliffs, N.Y.,1970.

12. R.H.Kingston, Detection of Optical and Infrared Radiation. In Springer Series in Optical Science, 10, Springer-Verlag, Berlin-Heidelberg-New York, 1978.

13. L.Okunev, Visha Algebra, Radyanska Shkola, Kiev, 1954.

14. V.M. Kostarchuk, B.I.Hazet, Kurs Vishei Algebri, Visha Shkola, Kiev, 1969.

15. N.B. Lukyanchikova, Noise Research in Semiconductor Physics, Gordon and Breach Sci. Publ. 1996. 